\title{
Chromosomal organization and fluorescence in situ hybridization of the human Sirtuin 6 gene
}

\author{
ULRICH MAHLKNECHT ${ }^{1}$, ANTHONY D. HO ${ }^{1}$ and SUSANNE VOELTER-MAHLKNECHT ${ }^{2}$ \\ ${ }^{1}$ Department of Hematology/Oncology, University of Heidelberg Medical Center, Im Neuenheimer Feld 410, \\ D-69120 Heidelberg; ${ }^{2}$ Department of Occupational, Social and Environmental Medicine, \\ University of Mainz, Obere Zahlbacher Str. 67, D-55131 Mainz, Germany
}

Received August 17, 2005; Accepted October 21, 2005

\begin{abstract}
Sirtuin 6 (SIRT6) is a member of the sirtuin deacetylases (sirtuins), which are derivatives of the yeast Silent information regulator 2 (Sir2) protein. SIR2 and its mammalian derivatives play a central role in epigenetic gene silencing, recombination, metabolism, cell differentiation and in the regulation of aging. In contrast to most sirtuins, SIRT6 lacks $\mathrm{NAD}^{+}$-dependent protein deacetylase activity. We have isolated and characterized the human Sirt6 genomic sequence, which spans a region of 8,427 bp and which has one single genomic locus. Determination of the exon-intron splice junctions found the full-length SIRT6 protein to consist of 8 exons ranging in size from $60 \mathrm{bp}$ (exon 4) to $838 \mathrm{bp}$ (exon 8 ). The human Sirt6 open reading frame encodes a 355 -aa protein with a predictive molecular weight of $39.1 \mathrm{kDa}$ and an isoelectric point of 9.12. Characterization of the 5' flanking genomic region, which precedes the Sirt6 open reading frame, revealed a TATA- and CCAAT-box less promoter with an approximately 300-bp long $\mathrm{CpG}$ island. A number of AML-1 and GATA-x transcription factor binding sites were found which remain to be further evaluated experimentally. Fluorescence in situ hybridization analysis localized the human Sirt6 gene to chromosome 19p13.3; a region which is frequently affected by chromosomal alterations in acute leukemia. Human SIRT6 appears to be most predominantly expressed in bone cells and in the ovaries while, in the bone marrow, it is practically absent. The functional characteristics of SIRT6 are essentially unknown at present and remain to be elucidated.
\end{abstract}

Correspondence to: Dr U. Mahlknecht, Department of Hematology/ Oncology, University of Heidelberg Medical Center, Im Neuenheimer Feld 410, D-69120 Heidelberg, Germany

E-mail: ulrich.mahlknecht@med.uni-heidelberg.de

Abbreviations: HDAC, histone deacetylase; HAT, histone acetyltransferase

Key words: sirtuins, histones, chromatin, histone deacetylase, chromosomes, genes, structural

\section{Introduction}

Based on structural and functional similarities, mammalian histone deacetylases are grouped into four categories, of which three contain non-sirtuin HDACs comprising the yeast histone deacetylases, RPD3 (class I HDACs), HDA1 (class II HDACs) and the more recently described HDAC11-related enzymes (class IV HDACs), while one category consists of sirtuin histone deacetylases (class III HDACs), which are homologs to the yeast Sir2 protein. SIRT1 is the mammalian sirtuin that is most closely related to S. cerevisiae SIR2. By contrast, mammalian SIRT6 is strongly related to SIRT7 (1) but only distantly homologous to human SIRT1 and yeast SIR2. The currently known seven human sirtuins have been further subgrouped into four distinct phylogenetic classes: SIRT1, SIRT2, and SIRT3 (subclass 1); SIRT4 (subclass 2); SIRT5 (subclass 3); and, finally, SIRT6 and SIRT7 (subclass 4, Fig. 1 and Table I) $(1,2)$. Derivatives of the yeast SIR2 histone deacetylase share a common catalytic domain which is highly conserved in organisms ranging from bacteria to humans and which is composed of two distinct motifs that bind $\mathrm{NAD}^{+}$and the acetyl-lysine substrate, respectively $(3,4)$. The yeast silent information regulator 2 protein (SIR2) is a $\mathrm{NAD}^{+}$-dependent histone deacetylase, which hydrolyzes one molecule of $\mathrm{NAD}^{+}$for every lysine residue that is deacetylated (5). The yeast Sir2 protein, as well as its mammalian derivatives, has been shown to directly modify chromatin and to silence transcription (6-10), to modulate the meiotic checkpoint (11) and, as a probable anti-aging effect, to increase genomic stability and suppress rDNA recombination $(8,12,13)$. While yeast SIR2 exclusively targets histone proteins, mammalian SIRT1 has a large and growing list of targets, such as p53 and forkhead transcription factors, which are mammalian homologs of the Daf-16 protein, a key regulator within the insulin signaling pathway $(8,14)$.

SIRT6 is a broadly expressed protein, which is predominantly found in the cell nucleus. In a tissue distribution analysis in the mouse embryo, SIRT6 reached peak levels at day E11, which further persisted into adulthood in muscle, brain and heart cells (15). The yeast silent information regulator 2 protein (SIR2); its mammalian orthologs, SIRT1, SIRT2, SIRT3, and SIRT5; and the bacterial protein, CobB; catalyze the tightly coupled cleavage of $\mathrm{NAD}^{+}$and protein deacetylation, producing nicotinamide and 2-O-acetyl-ADP- 


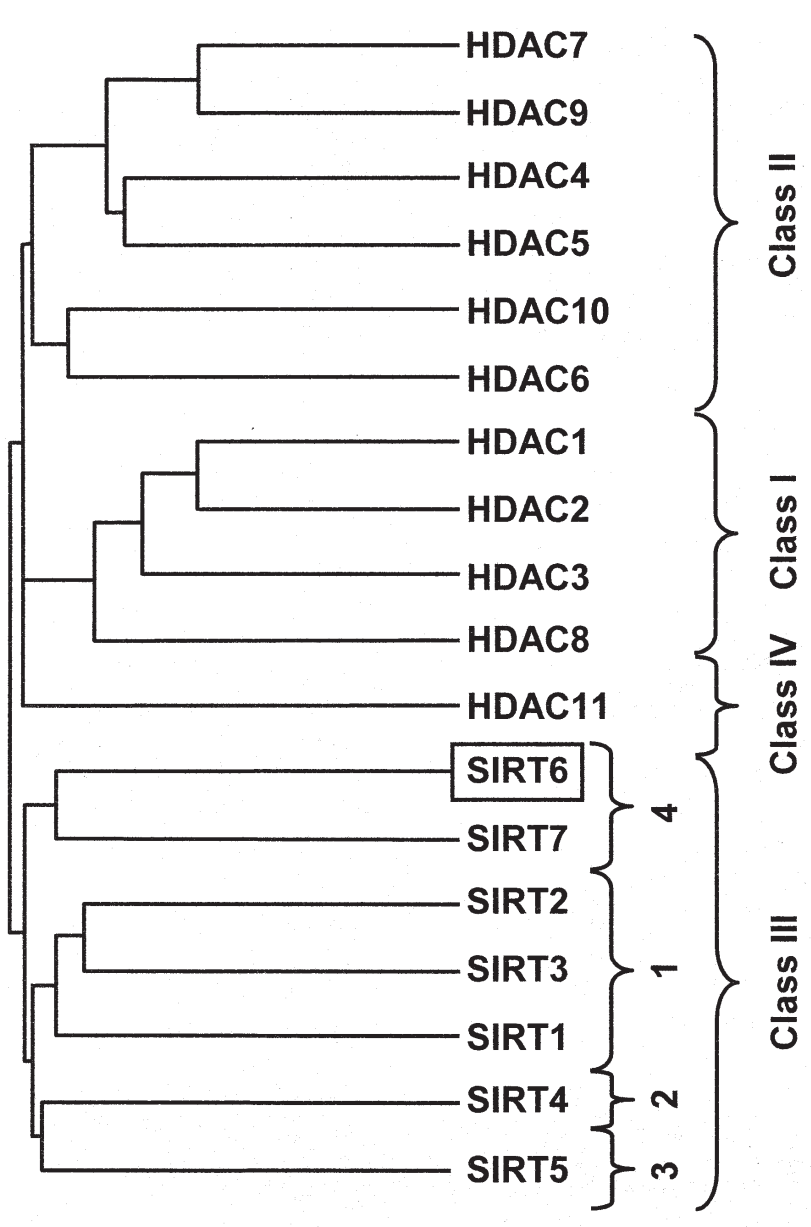

Figure 1. The position of human SIRT6 among the human orthologs for yeast RPD3, HDA1, SIR2 and HDAC11 related protein families of histone deacetylases (HDAC classes I-IV) and within the sirtuin-subclasses (subclasses 1-4) is shown (accession numbers of the sequences used in this tree: human HDAC1 (GenPept Q13547), human HDAC2 (GenPept Q92769), human HDAC3 (GenPept O15379), human HDAC8 (GenPept AAF73428), human HDAC4 (GenPept AAD29046), human HDAC5 (GenPept AAD29047), human HDAC6 (GenPept AAD29048), human HDAC7 (GenPept AAF04254), human HDAC9 (GenPept AAK66821), human HDAC10 (GenPept AAL30513), human HDAC11 (GenPept NP_079103), human SIRT1 (GenPept AAD40849), human SIRT2 (GenPept NP_036369), human SIRT3 (GenPept AAD40851), human SIRT4 (GenPept AAD40852), human SIRT5 (GenPept AAD40853), human SIRT6 (GenPept NP_057623) and human SIRT7 (GenPept AAF43431) (4). ribose reaction products (16). The deacetylase activity of sirtuin proteins is tightly coupled with their phosphoribosyltransferase activity and requires the presence of highly specific conserved amino-acid residues within the catalytic core of the protein, which are not contained in mammalian SIRT4, SIRT6, or SIRT7 and which are, therefore, lacking enzymatic deacetylase activity $(2,8,15,17-19)$.

Calorie restriction is known to induce a metabolic switch that increases the NAD/NADH ratio and/or decreases levels of nicotinamide, which is a yeast SIR2 inhibitor, and as a result activates SIR2 and increases rDNA stability $(8,20)$. Sirtuins, therefore, create a direct link between cellular energy status and longevity $(8,21)$. Mammalian SIRT1 binds, deacetylates and reduces the activity of several transcription factors in vivo, including MyoD, p53, and FOXO, thereby affecting cell differentiation and survival under stress $(14,22,23)$. The effect of SIRT1 on p53 may be inhibited by Nicotinamide (vitamin B3) (23-25). Calorie restriction in mammalian cells activates FOXO3A and increases FOXO3A-mediated expression of SIRT1, which depends on the presence of two p53 binding sites in the SIRT1 promoter, and a nutrient-sensitive physical interaction that was observed between FOXO3A and p53 $(14,26,27)$.

A cell that is low in energy will consume most of its NADH to generate ATP. The consequential high levels of nicotinamide adenine dinucleotide $\left(\mathrm{NAD}^{+}\right)$provide the indispensable cosubstrate for SIR2, which then activates acetyl-CoA synthetase (ACS) and subsequently results in the generation of more acetyl CoA, thus shunting more carbon into the NADH- and energy-generating TCA cycle (28). In addition to the generation of acetyl CoA, active Sir2 is also known to extend lifespan. More acetyl CoA for the citric acid cycle means more respiration, which has been associated with yeast lifespan extension when caloric intake is restricted. Accordingly, when mammals are short of food they also alter their metabolism such that both aging and reproduction are postponed until better times (28).

In contrast to SIRT1, only minimal information is currently available on human SIRT6, which is a distantly related ortholog of yeast SIR2 $(1,29)$, and which has been predicted to be predominantly a nuclear protein $(78.3 \%$ nuclear, $13.0 \%$ cyto-

Table I. Sequence identity and similarity among human class III sirtuin proteins. ${ }^{\text {a }}$

\begin{tabular}{|c|c|c|c|c|c|c|c|c|}
\hline & $\begin{array}{l}\text { Human } \\
\text { SIRT1 }\end{array}$ & $\begin{array}{c}\text { Human } \\
\text { SIRT2 }\end{array}$ & $\begin{array}{c}\text { Human } \\
\text { SIRT3 }\end{array}$ & $\begin{array}{c}\text { Human } \\
\text { SIRT4 }\end{array}$ & $\begin{array}{l}\text { Human } \\
\text { SIRT5 }\end{array}$ & $\begin{array}{c}\text { Human } \\
\text { SIRT6 }\end{array}$ & $\begin{array}{l}\text { Human } \\
\text { SIRT7 }\end{array}$ & $\begin{array}{l}\text { Yeast } \\
\text { SIR2 }\end{array}$ \\
\hline Human SIRT1 & & 42 & 40 & 30 & 28 & 22 & 23 & 40 \\
\hline Human SIRT2 & 65 & & 50 & 26 & 27 & 27 & 25 & 31 \\
\hline Human SIRT3 & 63 & 66 & & 28 & 31 & 28 & 28 & 35 \\
\hline Human SIRT4 & 47 & 43 & 43 & & 27 & 28 & 28 & 25 \\
\hline Human SIRT5 & 43 & 44 & 43 & 46 & & 21 & 24 & 26 \\
\hline Human SIRT6 & 39 & 44 & 40 & 43 & 36 & & 42 & 23 \\
\hline Human SIRT7 & 39 & 42 & 40 & 45 & 37 & 56 & & 21 \\
\hline Yeast SIR2 & 56 & 47 & 49 & 44 & 41 & 38 & 38 & \\
\hline
\end{tabular}

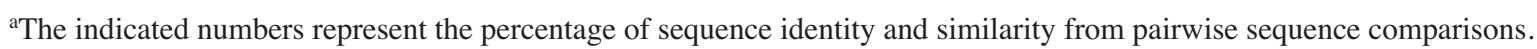


gccgggtggaatgttgagcacetgcettggaggaagctaggcagggcacaggccggcaagtgggaagtgegtgggtgaacgagtgcatgtgtgtgtcagggtacctgggta

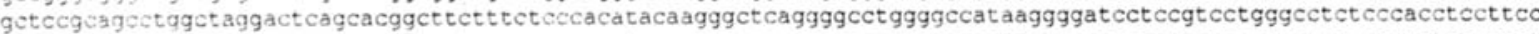

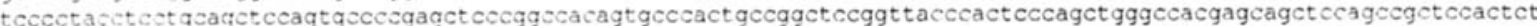

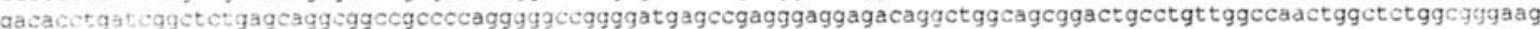

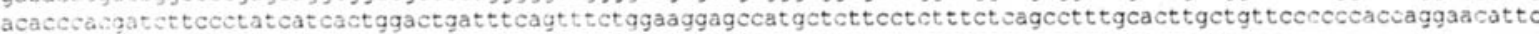

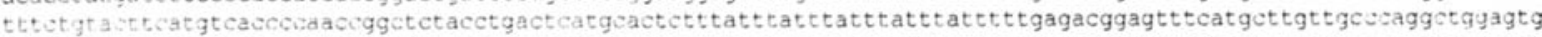
caatggeacgatctecggctcaccgcaatctccgcctcetgggttcaagcaattctectgectcagcetccegagcagctgcgattacaggecaccacacccagetaattt Lyf-1

tgtattttagtagagacggggtttctccatgttggtcagggtggtctcgaactcccaatctcaggtgatccacctgcettggcctcccaaagtgctgggattacaggtgt

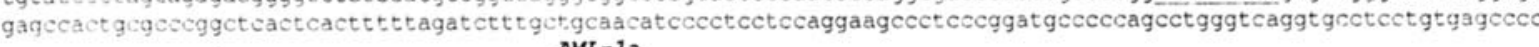
AML-1a

Ctcactgrectgtgcectctgcatcacccattgcaccccaaccacaccatgtcaccattacctggtcacatgtttgtgtccacccgcagccaccagggcatagatggtace acctttgetct zcagtgtgcccagtacaaagtaggtact caacacatgattcttaaatgagtectccaggccaccagecceattgaccttgagcaggacctgtcetgcaga Lyf-1

atgcagaucctccagttggatgatactcagataacttggccggacgcagtggctcacgcetgtaatcceagcactttgggaggccgaggcggtcagatcacctgaggtcag gagttcgggaccagcctgaccaacatggagaaaccecgtctctactaaaaatacaaaatttgctgggtgtggtggcgggcgcetgtaatcccagctactagggaggctg aggcaggagaatcgcttgaacccaagaggcggaggttgcagtgaaccgágattgcgccactgcactccagcctgggcaacagagcgaggctccgtcacacacacacacaca cacacacacacacacacaatgagatat caggtaactctg grggcattcaaacccttaggctggggtccetgctgtccggctctgtccttacggaatttactaatccg NFKB

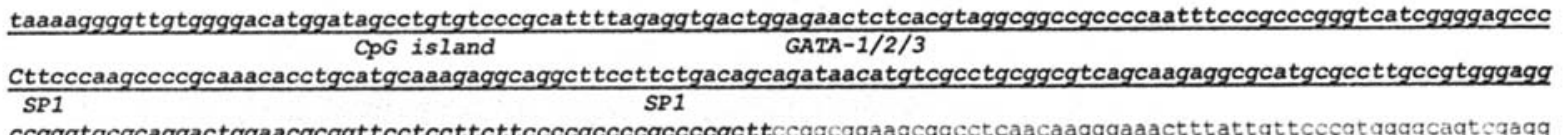

ccgggtgcgcaggactggaacgcggttcctccttcttccccgccccgcccogcttceggcggaagcggcetcaacaagggaaactttattgttccegtggggcagtegagg

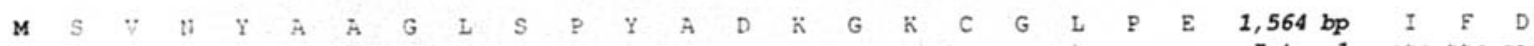
ATG teg gtg aat tac geg geg ggg ctg teg ceg tac geg gac aag ggc aag tge ggc ctc ceg gag ...Intron1.. atc ttc gac

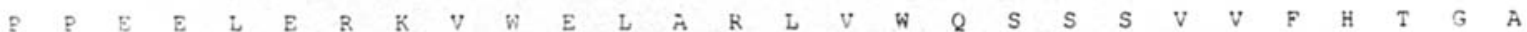
cec ceg gag gag ctg gag cgg aag gtg tgg gaa etg geg agg ctg gtc tgg cag tct tcc agt gtg gtg ttc cac acg ggt gcc

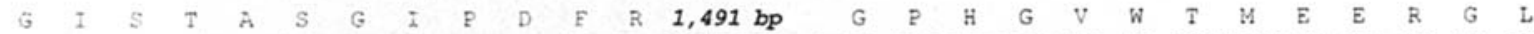
gge atc age act gec tet gge atc cec gac ttc ag ...Intron2... g ggt cce cac gga gte tgg ace atg gag gag cga ggt ctg

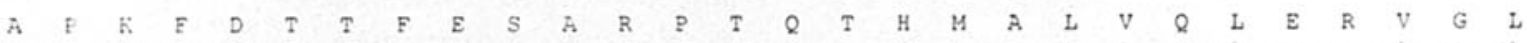
gec cee aag ttc gac ace acc ttt gag age geg egg cec acg cag acc cac atg geg ctg gtg cag ctg gag egc gtg gge ctc

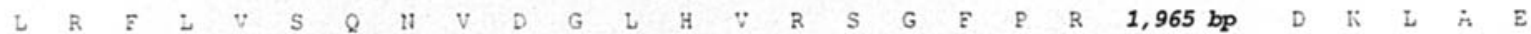
cte cge ttc ctg gtc age cag aac gtg gac ggg ctc cat gtg ege tca gge ttc cce ag ...Intron3... g gac aaa ctg gea gag

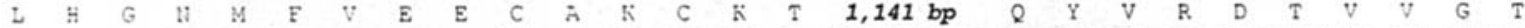
ctc cac ggg aac atg ttt gtg gaa gaa tgt gcc aag tgt aag ac ... Intron4.. g cag tac gtc cga gac aca gte gtg ggc acc

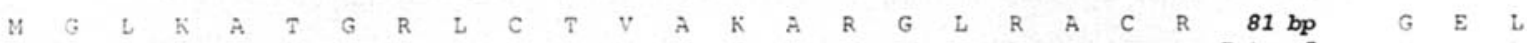
atg gge atg aag gce acg gge cgg ctc tge acc gtg get aag gea agg ggg ctg cga gec tgc ag ... Intron5.. g gga gag ctg

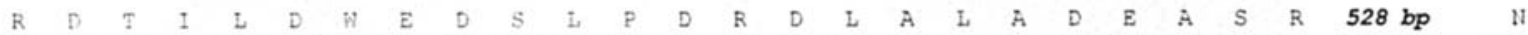
agg gac ace ate cta gac tgg gag gac tec ctg cec gac cgg gac ctg gea ctc gec gat gag gec age ag ...Intron6.. g aac

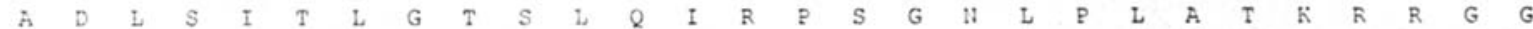
gec gac ctg tce atc acg ctg ggt aca teg ctg cag atc cgg cec age ggg aac ctg ceg ctg gct acc aag cge cgg gga ggc

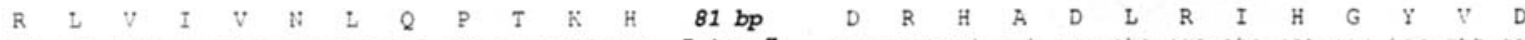
cgc etg gtc atc gtc aac etg cag cec acc aag cac ... Intron7... gac cgc cat get gac ctc cge atc cat gge tac gtt gac

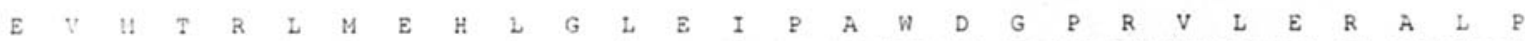
gág gte atg ace egg ctc atg gag cac ctg ggg ctg gag atc cec gec tgg gac gge cec cgt gtg ctg gaj agg geg ctg cea

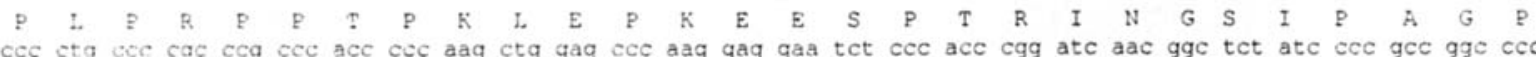

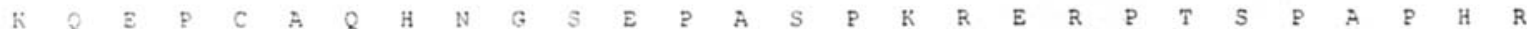
aag cag gaq cec tge gce cag cac aac gge tca gag cec gec agz cce aaa cgg gag egg cec acc age cet gce cec cac aga

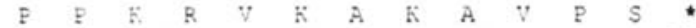
cec ece aaa agg gtg aag gec aag geg gtc ccc age TGA ceagggtgcttggggagggtggggcttttgtagaactgtggattcttttetetcg

tggt:etcacttgttacttgtttctgtccecgggagectcagggetctgagagetgtgctccaggceaggggttacacctgecetccgtggtcectccctgggetccagg gect ctggtgcggttecgggaagaagecacaccecagaggtgacagetgagecetgecacaccecagectctgacttgetgtgttgtcecagaggtgaggetgggecete

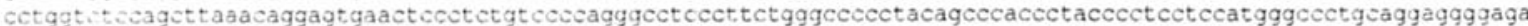
cccaccttgaggtgggggatcagtagaggettgeactgccttggggetggagggagacgtgggtccacaggettctggaaagtcctcaatgdataa aacaatttct ttettgcaus

Figure 2. The complete sequence of Sirt6 cDNA together with the predicted amino-acid sequence is shown with the location of each intron with respect to the cDNA sequence. The 1,638-bp human Sirt6 mRNA has an open reading frame of 1,065 bp that encodes the 355-aa SIRT6 protein. The untranslated 3' flanking region of exon 8 was shown to contain the eukaryotic polyadenylation consensus signal AATAAA (38), 484 bp downstream of the termination of translation signal TGA. Two kb of the 5' upstream promoter region are indicated $5^{\prime}$ upstream of the translational start codon. Putative transcription factor binding sites are entered and the corresponding DNA sequence is underlined. The CpG island is also underlined and the corresponding DNA sequence is indicated in lower-case bold italics. The translational start (ATG) and stop codons (TGA) are underlined, the polyadenylation signal (AATAAA) is boxed.

skeletal, $4.3 \%$ Golgi, $4.3 \%$ mitochondrial) (30). Human SIRT6 appears to be most predominantly expressed in bone cells and in the ovaries, while in the bone marrow it is practically absent. The further functional characterization of mammalian SIRT6 may help to further elucidate its potential role in the mediation of stress resistance, anti-apoptosis, anti-aging and 


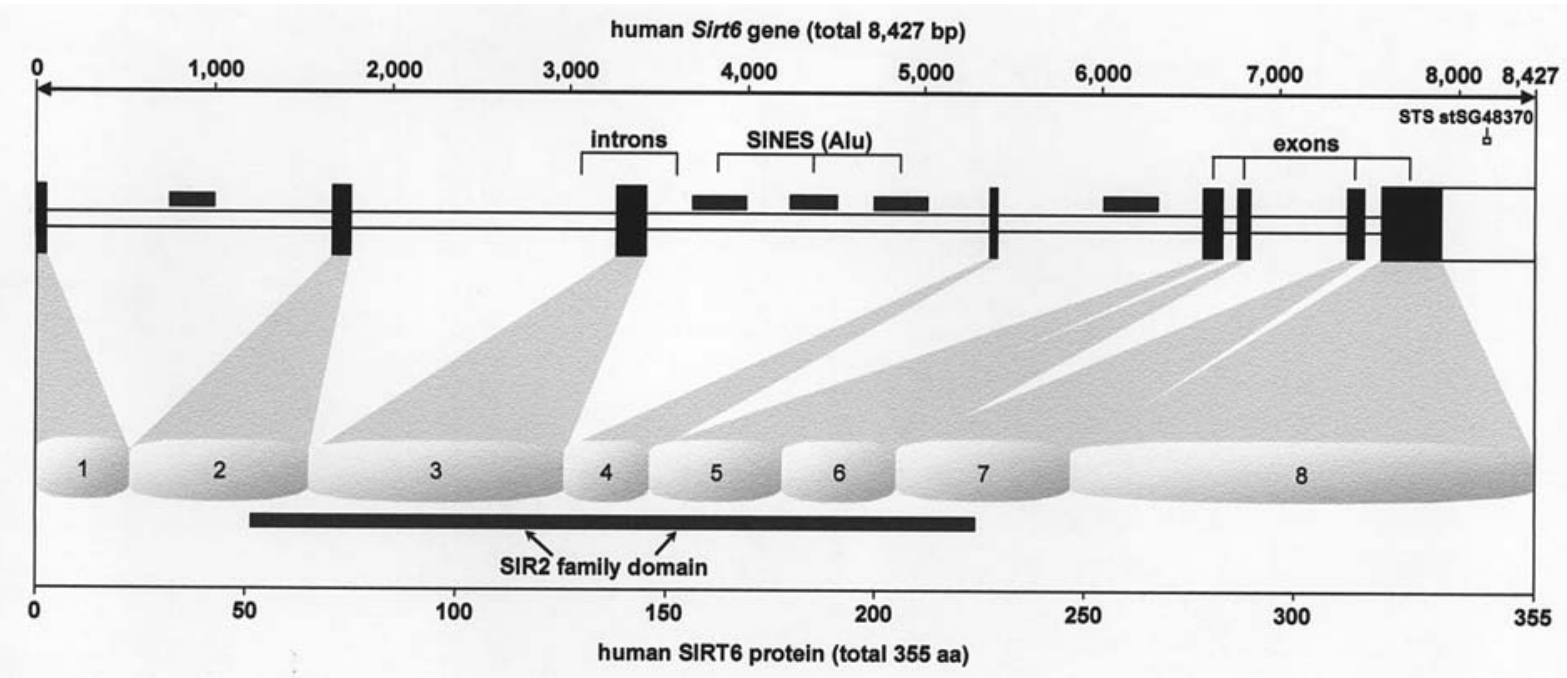

Figure 3. Genomic organization of the human Sirt6 gene. The genomic organization of the 8,427-bp long Sirt6 gene, which includes the relative position of exons and introns, is shown. Repetitive sequences, known as short interspersed nuclear elements (SINEs) are indicated. The position of internal STS-marker, stSG48370, is indicated. The sirtuin catalytic domain overlaps the SIRT6 protein region that is encoded by exons 2 through 7.

as a modulator of endocrine changes. In the study presented herein, we report the chromosomal localization and genomic organization of the human Sirt6 gene.

\section{Materials and methods}

Identification of the human Sirt6 cDNA. A homology search of the EST database at NCBI (National Center for Biotechnology Information) with the human Sirt6 cDNA that has been published earlier (1) yielded 7 positive cDNA clones of which one was obtained from the Reference Center of the German Human Genome Project (RZPD, Berlin, Germany). The authenticity of their inserts was confirmed by DNA cycle sequencing (Fig. 2).

Identification of BAC genomic clone, RZPDB737G031026D6. The human Sirt6 genomic clone was obtained from an arrayed BAC genomic library (Human Genomic Set - RZPD 1.0) after in silico screening with Sirt6 cDNA (GenBank clone NM_016539), which was shown to contain full-length human Sirt6 cDNA. BAC clone, RZPDB737G031026D6, was identified to contain inserts with an average size of approximately $120 \mathrm{~kb}$ in the vector, pBACe3.6, which included the human Sirt6 genomic sequence. BAC genomic DNA was prepared according to published protocols (31) and the Sirt6 insert was confirmed by cycle sequencing (32).

Instrumental methods. Dye terminator cycle sequencing was performed using the ABI PRISM ${ }^{\mathrm{TM}}$ BigDye Terminator Cycle Sequencing Ready Reaction Kit with AmpliTaq ${ }^{\mathrm{TM}}$ DNA polymerase (Perkin-Elmer, Branchburg, NJ) and analyzed using an ABI PRISM 310 Genetic Analyzer which utilizes the four-color sequencing chemistry.

PCR methods. The human Sirt6 sequence was partially sequenced by primer walking on both strands using a direct sequencing strategy (32). Sequencing reactions were performed using 0.6 $\mu \mathrm{g}$ cDNA and 20-30mer oligonucleotide primers
(Thermo Electron, Dreieich, Germany). Sequencing reactions were set up in a volume of $20 \mu \mathrm{l}$ containing $10 \mathrm{pmol}$ of the sequencing primer, $4 \mu 1$ BigDye Terminator Cycle Sequencing Ready Reaction Mix (Perkin-Elmer, Norwalk, CT), and DNA as indicated, and $\mathrm{ddH}_{2} \mathrm{O}$ was added to a final volume of $20 \mu \mathrm{l}$. The thermal cycling profile for the sequencing of the cDNAclones was as follows: denaturation at $95^{\circ} \mathrm{C}$ for $30 \mathrm{sec}$, annealing at $50^{\circ} \mathrm{C}$ for $15 \mathrm{sec}$, extension at $60^{\circ} \mathrm{C}$ for $4 \mathrm{~min}$ (25 cycles), and storage at $4^{\circ} \mathrm{C}$.

Sirt6 chromosomal localization by fluorescence in situ hybridization (FISH). Standard chromosome preparations were used from a human lymphoblastoid cell line. In order to remove excess cytoplasm, slides were treated with pepsin $(0.5 \mathrm{mg} / \mathrm{ml}$ in $0.01 \mathrm{M} \mathrm{HCl}, \mathrm{pH} 2.0)$ at $37^{\circ} \mathrm{C}$ for $40 \mathrm{~min}$. Slides were then washed for $2 \times 10$ min in $1 \mathrm{X}$ PBS and $1 \times 10 \mathrm{~min}$ in $1 \mathrm{X} \mathrm{PBS} / 50 \mathrm{mM} \mathrm{MgCl}_{2}$ at room temperature. BAC DNA was labeled by using a standard nick translation procedure. Digoxigenin (Roche Diagnostics) was used as labeled dUTP at the concentration of $40 \mu \mathrm{M}$. Probe length was analyzed on a $1 \%$ agarose gel. The probe showed an optimal average length of $\sim 300$ bp after nick translation. Approximately $50 \mathrm{ng}$ DNA were pooled with $2 \mu \mathrm{g}$ cot- 1 in $10 \mu$ l hybridization buffer (50\% formamide, 2 X SSC, $10 \%$ dextran sulfate). The DNA was applied to chromosomes fixed on a slide, mounted with a cover slip and sealed with rubber cement. Probe DNA and chromosomes were denatured at $72^{\circ} \mathrm{C}$ for $3 \mathrm{~min}$. Hybridization was overnight at $37^{\circ} \mathrm{C}$ in a wet chamber. After hybridization, the cover slip was carefully removed and the slide was washed in $2 \mathrm{X}$ SSC for $8 \mathrm{~min}$. The slide was then incubated at $72^{\circ} \mathrm{C}$ in $0.4 \mathrm{X} \mathrm{SSC} / 0.1 \%$ Tween for $1 \mathrm{~min}$, washed shortly in $2 \mathrm{X} \mathrm{SSC}$ at room temperature, and stained in DAPI (4',6-diamidino-2-phenylindole) for $10 \mathrm{~min}$. For microscopy, the slide was mounted in antifade solution (Vectashield). In situ hybridization signals were analyzed on a Zeiss Axioplan II microscope. Each image plain (blue and orange) was recorded separately with a b/w CCD camera. Chromosomes and FISH signals were then displayed in false colors 


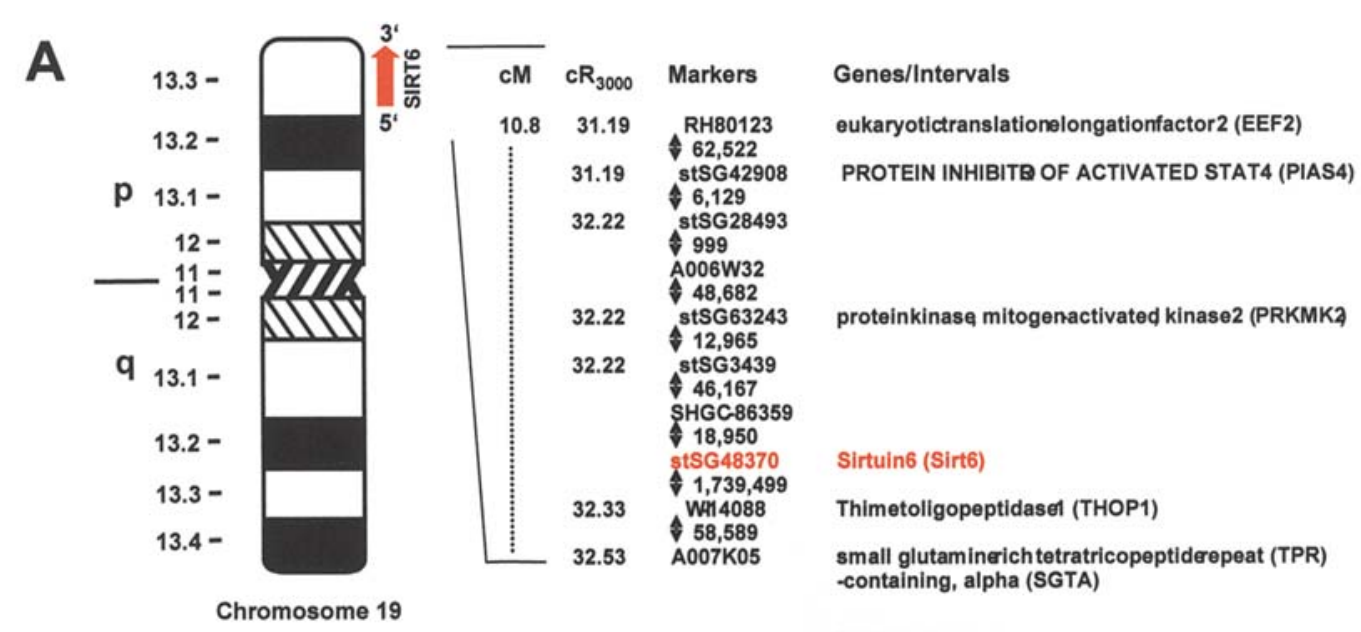

B
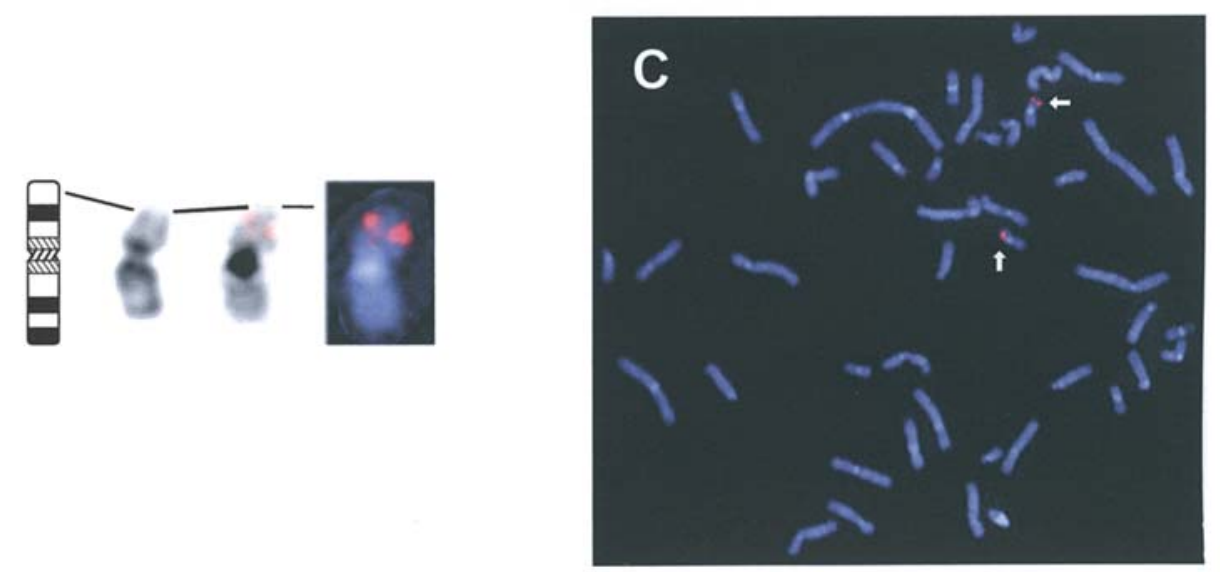

Figure 4. Chromosomal mapping of the human Sirt6 gene. Upper panel (A), chromosome 19 idiogram according to the International System for Cytogenetic Nomenclature (ISCN 1995). The chromosomal position of BAC clone RZPDB737G031026D6 in close proximity to the PRKMK2 gene (located telomerically) and the THOP1 gene (located centromerically) is shown as well as the chromosomal orientation of Sirt6 gene (red arrow). (B) From left to right, next to the chromosome 19 idiogram, pictures of a DAPI-stained chromosome 19, together with the same chromosome carrying the BAC hybridization signal, are illustrated. (C) Fluorescence in situ hybridization of BAC clone RZPDB737G031026D6 to human chromosome 19p13.3.

and images merged on the computer. Camera control, image capture and merging were performed using SmartCapture X software (Digital Scientific, Cambridge, UK) (Fig. 4).

Sequence analysis and computer database searches. DNA sequence analysis was performed using the HUSAR (Heidelberg Unix Sequence Analysis Resources) server hosted by the Biocomputing Service Group at the German Cancer Research Center (DKFZ, Heidelberg) and the UniGene and LocusLink programs at the National Center for Biotechnology Information (NCBI). Sequence comparisons were performed using the BLAST algorithm of the GenBank and EMBL databases (33). Protein similarity scores were calculated from fast alignments generated by the method of Wilbur and Lipman using the CLUSTAL W Multiple Alignment Program Version 1.7 (Figs. 1 and 5; Tables I and III) (34). Protein motifs were identified online at the ExPASy (Expert Protein Analysis System) proteomics server of the Swiss Institute of Bioinformatics (SIB) with the program, PROSITE, and double-checked using the MotifFinder program hosted by the GenomeNet server at the Bioinformatics Center at the Institute for Chemical Research from the Kyoto University (Japan). Potential transcription factor binding sites were identified using the TRANSFAC program, which is part of the GenomeNet Computation Service (see above), but remain to be confirmed experimentally. Sequence similarities were calculated using GAP software, which considers all possible alignments and gap positions between two sequences and creates a global alignment that maximizes the number of matched residues and minimizes the number and size of gaps on the HUSAR server (35). Repetitive and $\mathrm{CpG}$ elements were identified on the RepeatMasker Server and with the CPG software hosted by the European Bioinformatics Institute (EMBL outstation) (Figs. 2 and 3).

Phylogenetic analysis. Phylogenetic trees were constructed from known human class I through class IV histone deacetylase sequences which were obtained from protein sequence similarity searches with the yeast proteins, RPD3, HDA1 and SIR2, using the BLAST 2.0 program at NCBI database (Nonredundant GenBank CDS: translations+PDB + Swiss Prot + SPupdate+PIR). Progressive multiple sequence alignments were performed using the CLUSTAL W Multiple Alignment Program Version 1.7 (Fig. 1) (4,36). Trees were then calculated and drawn using PileUp software, which computes a multiple sequence alignment using a simplification of the progressive alignment method of Feng and Doolittle (37) and which can plot a dendrogram like the one below, that shows the clustering relationships used to determine the order of the pairwise alignments that together create the final multiple sequence 


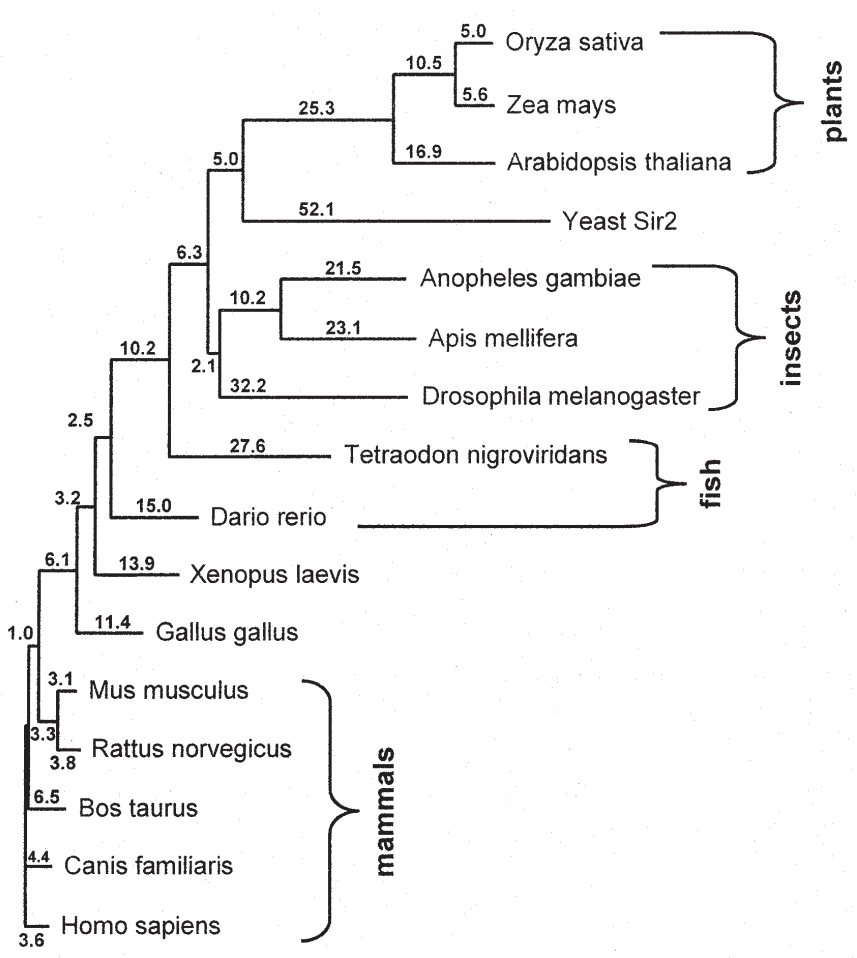

Figure 5. SIRT6 phylogenetic tree. This dendrogram depicts the sequence relatedness of the human SIRT6 protein with the SIRT6 homologs from different species. The GenPept accession numbers correspond to the ones that have also been used for the multiple sequence alignment as shown in Table III.

alignment. Distance along the vertical axis is proportional to the difference between sequences; distance along the horizontal axis has no significance at all. Trees in Fig. 5 were calculated and drawn using PATH (Phylogenetic Analysis Task in HUSAR) software, which estimates and realizes phylogenies by executing the three main phylogenetic methods: distance, parsimony and maximum likelihood, and which is hosted by the HUSAR (Heidelberg Unix Sequence Analysis Resources) server from the Biocomputing Service Group at the German Cancer Research Center (DKFZ, Heidelberg).

\section{Results}

Identification and cloning of cDNAs encoding human Sirt6. Homology searches of the dbEST at NCBI (National Center for Biotechnology Information) (33) for the Sir6 cDNA sequence $(1,2)$ yielded 7 positive cDNA clones: GenBank NM_016539 (1,638 bp), AF233396 (1,638 bp), AK074810 (1,603 bp), BC004218 (1,398 bp), BC005026 (1,600 bp), BC028220 (1,585 bp) and CR457200 (1,068 bp), of which GenBank clone NM_016539 was obtained from the Reference Center of the German Human Genome Project (RZPD, Berlin, Germany). The authenticity of its insert was confirmed by DNA cycle sequencing (Fig. 2). Sequences flanking the 5' and 3 ' ends of the Sirt6 open reading frame were identified from the Sirt6 human genomic clone, BAC RZPDB737G031026D6. The human Sirt6 mRNA is transcribed into a 1,638-bp mRNA with an open reading frame of $1,065 \mathrm{bp}$, which is translated into a 355-aa protein with a predictive molecular weight of $39.1 \mathrm{kDa}$ and an isoelectric point of 9.12. Characterization of the 5 ' flanking genomic region, which precedes the Sirt6 open reading frame, revealed a TATA- and CCAAT-box less promoter with an approximately 300-bp CpG island. A number of GATA-x and AML-1 transcription factor binding sites were found which remain to be further evaluated experimentally. Fluorescence in situ hybridization analysis localized the human Sirt6 gene to chromosome 19p13.3. Translational stop codons in all reading frames precede the human Sirt6 open reading frame. The 3' flanking region was shown to contain the eukaryotic polyadenylation consensus signal AATAAA (38), $484 \mathrm{bp}$ downstream of the termination of translation signal TGA (Fig. 2).

Identification and characterization of the human Sirt6 genomic locus. The human Sirt6 genomic clone was obtained from an arrayed BAC genomic library (Human Genomic Set - RZPD 1.0) after in silico screening with Sirt6 cDNA (GenBank clone NM_016539), which was shown to contain full-length human Sirt6 cDNA. BAC clone RZPDB737G031026D6 was identified to contain inserts with an average size of approximately $120 \mathrm{~kb}$ in the $11.6-\mathrm{kb}$ vector, pBACe3.6, which

Table II. Exon/intron splice-junctions of the human Sirt6 gene: exon sequences are given in uppercase and intron sequences are given in lowercase letters. ${ }^{\text {a }}$

\begin{tabular}{|c|c|c|c|c|c|}
\hline $\begin{array}{l}\text { Exon } \\
\text { no. }\end{array}$ & $\begin{array}{c}\text { Exon } \\
\text { size }\end{array}$ & 5'-splice donor & $\begin{array}{c}\text { Intron } \\
\text { no. }\end{array}$ & $\begin{array}{l}\text { Intron } \\
\text { size }\end{array}$ & 3'-splice acceptor \\
\hline 1 & 66 & ССТCCCGGAGgtgagcgegtct & 1 & 1.564 & ctccсcсcacagATCTTCGACC \\
\hline 2 & 128 & CCGACTTCAGgtctgtgattgt & 2 & 1.491 & tgccaccttcagGGGTCCCCAC \\
\hline 3 & 183 & GCTTCCCCAGgtaacaccctgg & 3 & 1.965 & ctcttcccacagGGACAAACTG \\
\hline 4 & 60 & AGTGTAAGACgtgagtgccacc & 4 & 1.141 & tccctgacacagGCAGTACGTC \\
\hline 5 & 96 & GAGCCTGCAGgtgagcсассCC & 5 & 81 & tcctcattgcagGGGAGAGCTG \\
\hline 6 & 81 & AGGCCAGCAGgtctgacCCCCC & 6 & 528 & CCCCggccecagGAACGCCGAC \\
\hline 7 & 124 & CACCAAGCACgtaggtgtctga & 7 & 81 & gCCCCCCggcagGACCGCCATG \\
\hline 8 & 838 & & & & \\
\hline
\end{tabular}

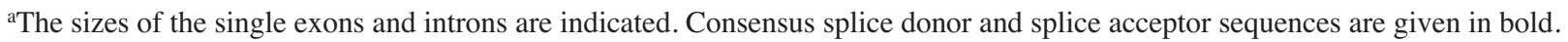




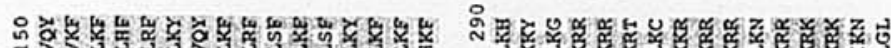

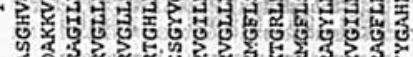

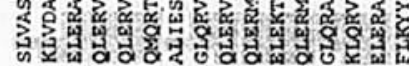

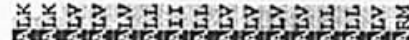
What

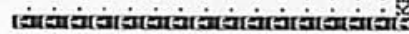

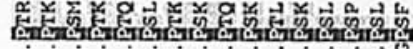
:!!!!!!!!

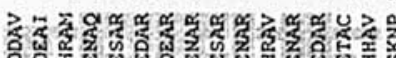

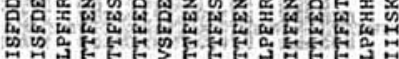

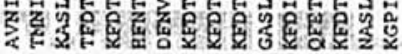

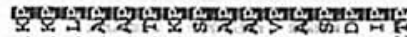

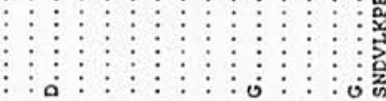

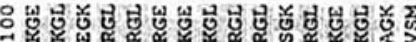

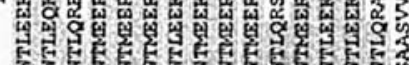

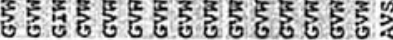

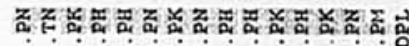

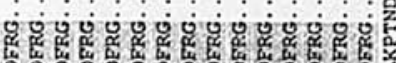

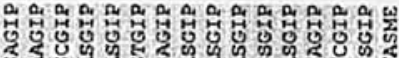

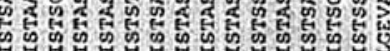
हैं

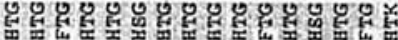

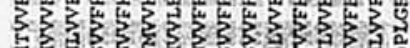

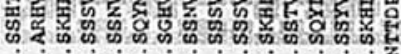

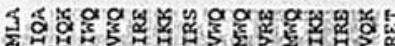

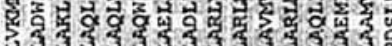

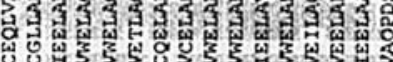

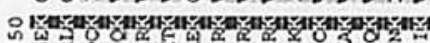

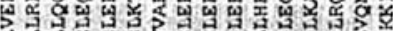

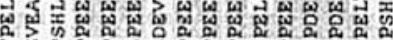

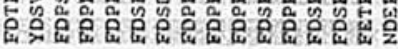
W $>$ H

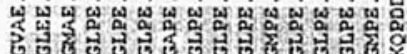

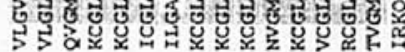
(1)

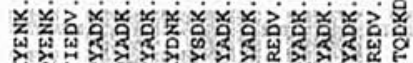

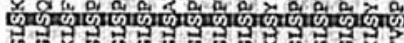

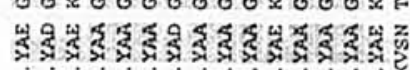

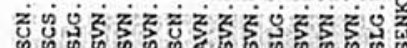
च?

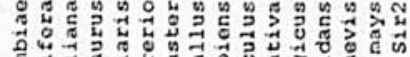

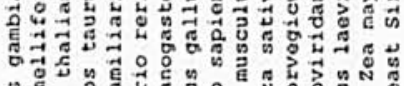

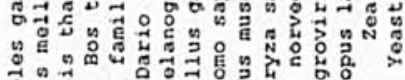

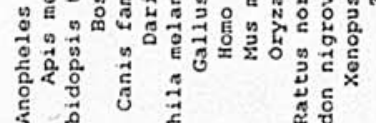

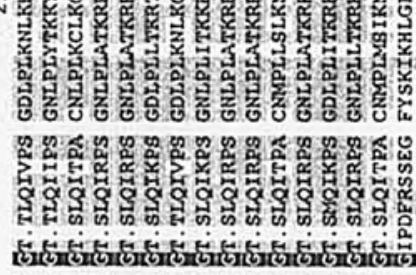

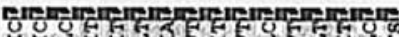

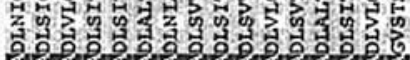
3.

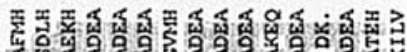

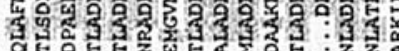

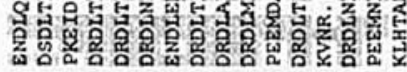

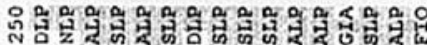

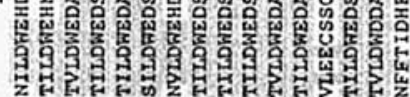

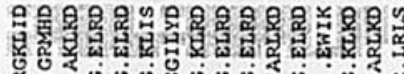

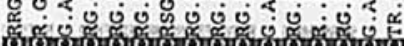

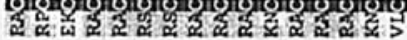
虽品: :

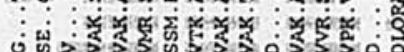

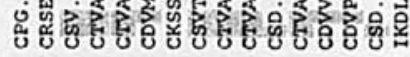

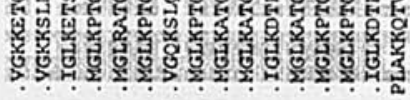

tyำำ

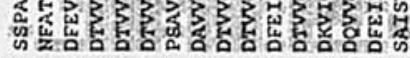

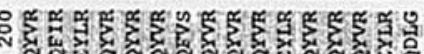

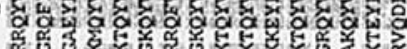

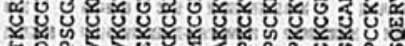

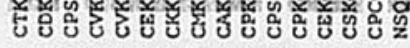

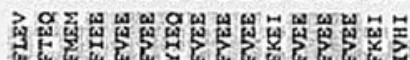

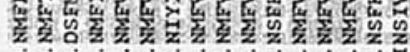

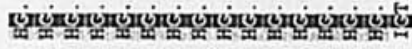

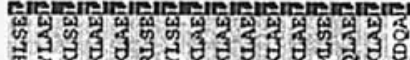

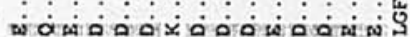

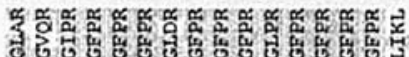

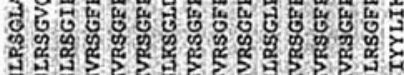

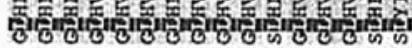

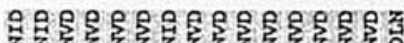

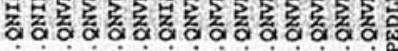

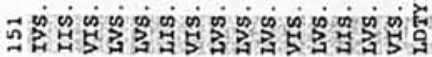

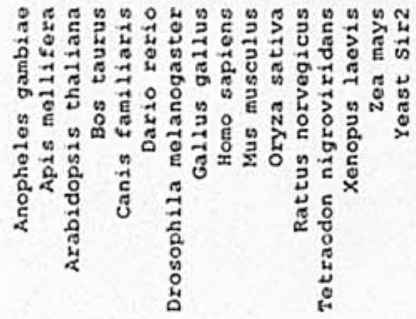

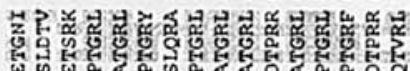

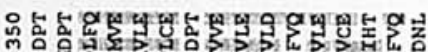

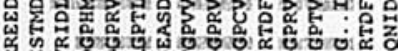

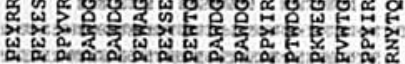

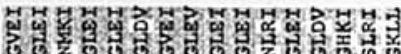

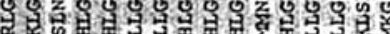

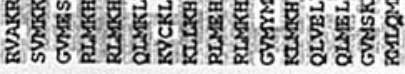

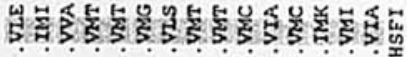

ล่แน

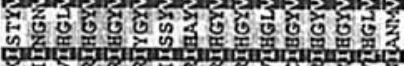

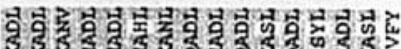

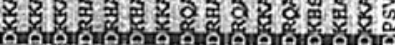

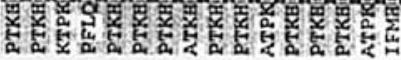

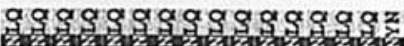
voth thenth

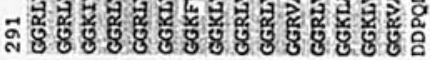

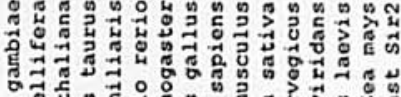

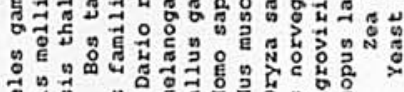

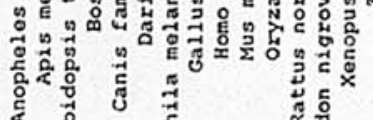

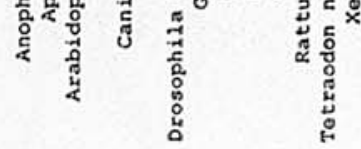

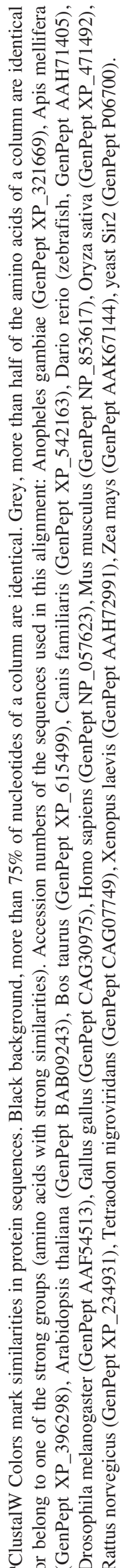




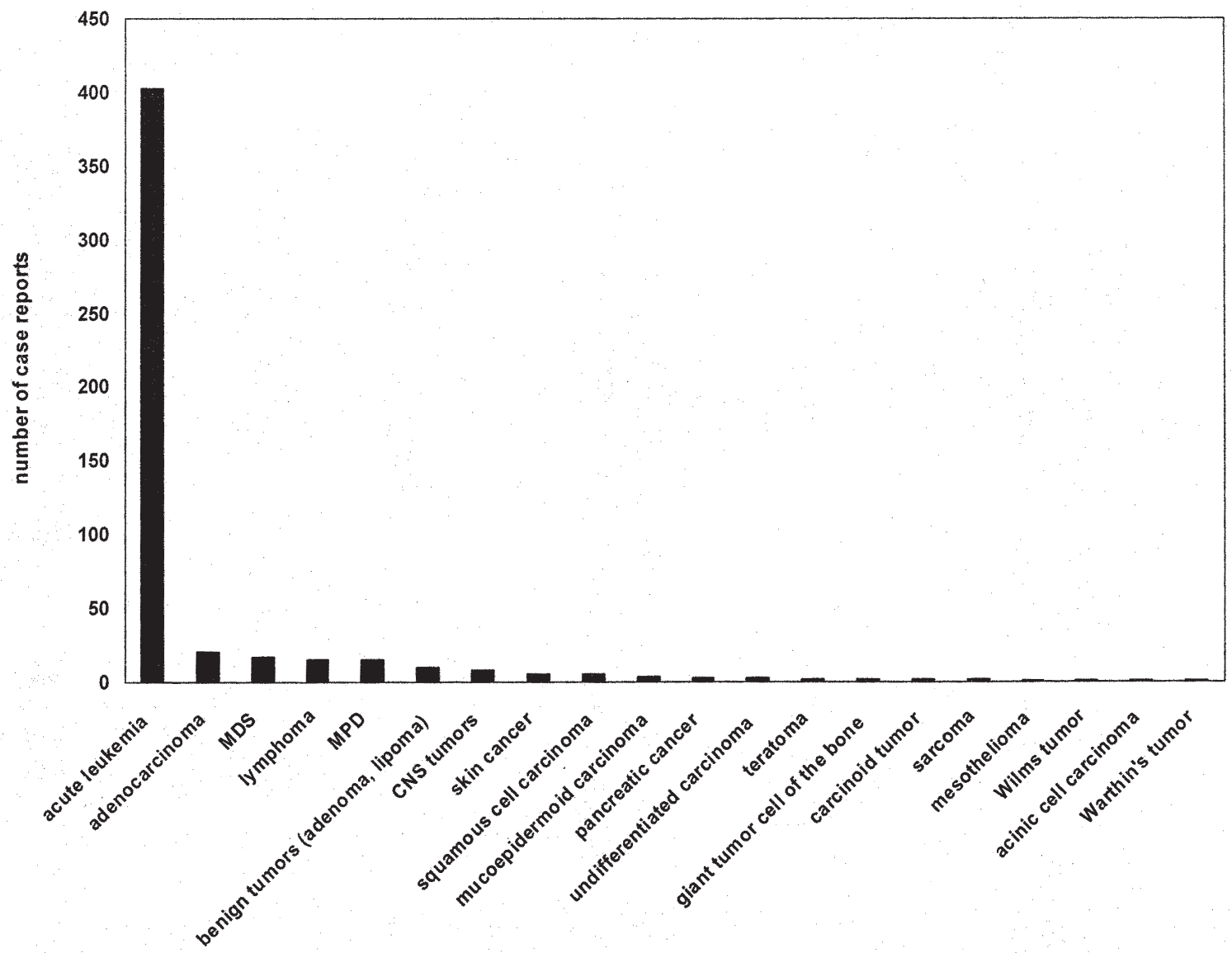

Figure 6. The chromosome region, 19p13, is a section which has been found to be involved in various malignancies. Of 522 case reports, which have been retrieved from the Cancer Genome Anatomy Project (CGAP) database at the National Cancer Institute (45), the most frequent mutations and chromosomal alterations have been found in acute leukemias (403; with 135 AML, 257 ALL, 11 bilineage biphenotypic leukemia), adenocarcinomas (21), MDS (17), lymphoma (15; with 4 CLL, 1 mycosis fungoides, 3 Burkitt lymphoma, 1 Hodgkin's disease, 2 multiple myeloma, 1 T-cell lymphoma, 1 B-cell lymphoma, 2 hairy cell leukemia), myeloproliferative disease (15; with 14 CML, 1 polycythaemia vera), benign tumors (10; with 2 lipoma, 2 papilloma, 2 adenoma, 2 leiomyoma, 1 solitary fibrous tumor, 1 benign epithelial tumor), central nervous system tumors (9; with 7 meningiomas, 1 astrocytoma, 1 primitive neuroectodermal tumor), skin cancer (5; with 4 basal cell carcinoma, 1 dysplastic nevus), 5 squamous cell carcinomas, 4 mucoepidermoid carcinomas, 3 pancreatic cancers, 3 undifferentiated carcinomas, 2 teratomas, 2 giant tumor cell of the bone, 2 carcinoid tumors, 2 sarcomas, 1 mesothelioma, 1 Wilms tumor, 1 acinic cell carcinoma and 1 Warthin's tumor.

included the human Sirt6 genomic sequence. BAC genomic DNA was prepared according to published protocols (31) and the Sirt6 insert was confirmed by cycle sequencing (32). Genomic sequence comparison analyses with the BLAST algorithm helped us with the identification of human chromosome 6 genomic contig NT_011245, which was sequenced and assembled from individual clone sequences by the Human Genome Sequencing Consortium together with NCBI. We have used this sequence for the determination of Sirt6 introns and exon/intron boundaries (Table II). The human Sirt6 gene spans a region of 8,427 bp (Fig. 3). Determination of the exon-intron splice junctions found the full-length SIRT6 protein to consist of 8 exons ranging in size from $60 \mathrm{bp}$ (exon 4 ) to $838 \mathrm{bp}$ (exon 8 ). Within introns 1,3 and 4 we identified an accumulation of interspersed repetitive elements, SINEs (short interspersed nuclear elements) (Fig. 3). Additionally, we have identified an internal STS-marker, sTSG48370, which is located within the untranslated proportion of exon 8 . The sirtuin catalytic domain, which is highly conserved in all members of mammalian sirtuins that have been described so far as well as in their Sir2 yeast ancestor protein, is found between amino-acid residues 52 and 221, i.e. within exons 2 and 7 of the protein (Fig. 3).
Sirt6 is a single copy gene. Sequencing and results obtained by electronic PCR of BAC clone RZPDB737G031026D6 identified STS-marker sTSG48370 to be located within the Sirt6 genomic sequence. These data, together with the results obtained by electronic PCR and the reported location of the mentioned STS-markers, indicated one single site of hybridization of Sirt6 on human metaphase chromosomes and its specific localization on chromosome 19p13.3 (Fig. 4).

Sirt6 expression analyses. In silico expression profile analyses were carried out using the UniGene EST profile viewer, which is hosted by the NCBI homepage, and the Human GeneAtlas Gene Expression Database, which is hosted by the Genomics Institute of the Novartis Research Foundation (GNF) and which identified human SIRT6 to be most predominantly expressed in bone cells and in the ovaries but practically absent in bone marrow (39-42).

Phylogenetic analysis and pairwise sequence comparisons. We have screened the 'all non-redundant GenBank CDS translations + RefSeq Proteins + PDB + SwissProt + PIR + $\mathrm{PRF}$ expressed sequence tag database' ('nr' at NCBI) with 
the human SIRT6 protein sequence and identified several yeast and human histone deacetylases which were sharing a significant degree of sequence homology with human SIRT6, indicating a high degree of phylogenetic conservation of protein structure and associated function throughout evolution. The tree was constructed after bootstrapping and depicts a subdivision into four main evolutionary branches (plants, insects, fish and mammals) (Fig. 5). In an additional analysis, a consensus evolutionary tree was obtained for class I through class IV human sirtuin and non-sirtuin HDACs on the basis of an alignment of the yeast RPD3, HDA1, SIR2 and HDAC11 human homologous proteins (Fig. 1). Obviously, the sirtuin family of HDACs (class III) does not reveal significant sequence homology with the three classes of non-sirtuin HDACs. The tree was constructed after bootstrapping and clearly identifies four families of human histone deacetylases with HDAC1, HDAC2, HDAC3 and HDAC8 being members of the yeast RPD3 family of histone deacetylases (so-called 'mammalian class I histone deacetylases'), HDAC4, HDAC5, HDAC6, HDAC7, HDAC9 and HDAC10 being members of the yeast HDA1 family of histone deacetylases (mammalian class II histone deacetylases) and SIRT1 through SIRT7 being homologs of the yeast SIR2 protein (mammalian class III histone deacetylases), while HDAC11 is so far the only member of a distinct group of class IV HDACs (Fig. 1) (4).

\section{Discussion}

The human Sirt6 gene encodes members of the sirtuin family of proteins which are referred to as class III NAD ${ }^{+}$-dependent histone deacetylases on the basis of their homology to the yeast Sir2 protein (5). The members of the sirtuin family are characterized by a sirtuin core domain and are grouped into four subclasses with SIRT6 being a class 4 sirtuin member (Fig. 1). For most of the currently known human sirtuins, a function has not yet been determined. In yeast, however, sirtuin proteins are known to regulate epigenetic gene silencing and suppress recombination of rDNA. In addition to their deacetylating activity, human sirtuins may function as intracellular regulatory proteins with mono-ADP-ribosyltransferase activity (2). Human Sirt6 has been predicted to be predominantly a nuclear protein (30), which seems to be strongly expressed in bone cells and in the ovaries, while being practically absent in bone marrow (39-42). In the mouse embryo, SIRT6 has been reported to reach peak levels at day E11, which further persisted into adulthood in muscle, brain and heart cells (15).

In the present study, we report the identification, cloning and mapping of Sirt6 on the genomic level. Human Sirt6 is a single-copy gene that spans a region of approximately $8.5 \mathrm{~kb}$. It is composed of 8 exons (Fig. 3 and Table II) ranging in size from $60 \mathrm{bp}$ (exon 4) to $838 \mathrm{bp}$ (exon 8) and reveals an accumulation of interspersed SINEs (Alu repeats) within introns 1,3 , and 4 (43). The SIR2 family domain is highly conserved within all members of mammalian sirtuin proteins that have been described so far and is located within exons 2 through 7 (Fig. 3). The 5' upstream Sirt6 promoter region was found to contain a small $300 \mathrm{bp} \mathrm{CpG}$ island and lacks the canonical TATA-and CCAAT boxes (Fig. 2). TATA- independent transcription in the presence of accumulated $\mathrm{CpG}$ elements has been described to be a typical feature of constitutively active housekeeping genes (44). Human Sirt6 mRNA encodes a 355-aa protein with a predictive molecular weight of $39.1 \mathrm{kDa}$. Fluorescence in situ hybridization analysis in conjunction with electronic PCR localized the human Sirt6 gene to the sub-band of chromosome 19p13.3 (Fig. 4), a region which has been found to be involved in numerous chromosomal abnormalities in association with malignant disease, especially in acute leukemias (403 of 522 cases) (Fig. 6). These data have been retrieved from the Cancer Genome Anatomy Project (CGAP) database at the National Cancer Institute (45).

It is currently not clear to what extent chromosomal abnormalities that involve the chromosome 19p13 chromosomal region have an influence on SIRT6-mediated functional effects. It is, however, evident that a number of sirtuin proteins are located within chromosomal regions that are particularly prone to chromosomal breaks. In such cases, gains and losses of chromosomal material may affect the availability of functionally active sirtuin proteins, which in turn disturbs the tightly controlled intracellular equilibrium of protein acetylation and/or ADP ribosylation, respectively (46). Protein acetylation modifiers are therefore gaining increasing attention as potential targets in the treatment of cancer. Relaxation of the chromatin fiber facilitates transcription and is regulated by two competing enzymatic activities, histone acetyltransferases (HATs) and histone deacetylases (HDACs), which modify the acetylation state of histone proteins and other promoter-bound transcription factors. While HATs, which are frequently part of multisubunit coactivator complexes, lead to the relaxation of chromatin structure and transcriptional activation, HDACs tend to associate with multisubunit corepressor complexes, which results in chromatin condensation and the transcriptional repression of specific target genes.

Unfortunately, it is currently not possible to assess to what extent human SIRT6 is playing a role in the pathogenesis of hematological malignancies and acute myeloid leukemia in particular. It is, however, evident that SIRT6 contains multiple repetitive elements at the genomic level, which makes the region particularly prone to chromosomal breaks, while it is located within a chromosomal region that is known to be frequently part of chromosomal alterations in acute leukemia. In the context of such chromosomal modifications that may involve SIRT6, the SIRT6 protein could potentially be either missing, dysfunctional or exhibit its enzymatic activity at wrong times in the wrong places and therefore contribute to an imbalance of the intracellular acetylation status and to the development of disease. The further characterization of the functional role of human SIRT6 is therefore likely to become an exciting endeavor.

\section{Acknowledgements}

This work was supported by the German National Science Foundation (Deutsche Forschungsgemeinschaft, MA 2057/2-4).

\section{References}

1. Frye RA: Phylogenetic classification of prokaryotic and eukaryotic Sir2-like proteins. Biochem Biophys Res Commun 273: 793-798, 2000 
2. Frye RA: Characterization of five human cDNAs with homology to the yeast SIR2 gene: Sir2-like proteins (sirtuins) metabolize NAD and may have protein ADP-ribosyltransferase activity. Biochem Biophys Res Commun 260: 273-279, 1999.

3. Brachmann CB, Sherman JM, Devine SE, Cameron EE, Pillus L and Boeke JD: The SIR2 gene family, conserved from bacteria to humans, functions in silencing, cell cycle progression, and chromosome stability. Genes Dev 9: 2888-2902, 1995.

4. Voelter-Mahlknecht S, Ho AD and Mahlknecht U: FISH-mapping and genomic organization of the NAD-dependent histone deacetylase gene, Sirtuin 2 (Sirt2). Int J Oncol 27: 1187-1196, 2005.

5. Imai S, Armstrong CM, Kaeberlein M and Guarente L: Transcriptional silencing and longevity protein Sir2 is an NADdependent histone deacetylase. Nature 403: 795-800, 2000.

6. Vaquero A, Scher M, Lee D, Erdjument-Bromage H, Tempst $\mathrm{P}$ and Reinberg D: Human SirT1 interacts with histone H1 and promotes formation of facultative heterochromatin. Mol Cell 16: 93-105, 2004

7. Blander G, Olejnik J, Krzymanska-Olejnik E, McDonagh T, Haigis M, Yaffe MB, et al: SIRT1 shows no substrate specificity in vitro. J Biol Chem 280: 9780-9785, 2005.

8. Blander G and Guarente L: The Sir2 family of protein deacetylases. Annu Rev Biochem 73: 417-435, 2004.

9. Straight AF, Shou W, Dowd GJ, Turck CW, Deshaies RJ, Johnson AD, et al: Net1, a Sir2-associated nucleolar protein required for rDNA silencing and nucleolar integrity. Cell 97: 245-256, 1999.

10. Fritze CE, Verschueren K, Strich R and Easton Esposito R: Direct evidence for SIR2 modulation of chromatin structure in yeast rDNA. EMBO J 16: 6495-6509, 1997.

11. San-Segundo PA and Roeder GS: Pch2 links chromatin silencing to meiotic checkpoint control. Cell 97: 313-324, 1999.

12. Kaeberlein M, McVey M and Guarente L: The SIR2/3/4 complex and SIR2 alone promote longevity in Saccharomyces cerevisiae by two different mechanisms. Genes Dev 13: 2570-2580, 1999.

13. Shore D, Squire M and Nasmyth KA: Characterization of two genes required for the position-effect control of yeast matingtype genes. EMBO J 3: 2817-2823, 1984.

14. Brunet A, Sweeney LB, Sturgill JF, Chua KF, Greer PL, Lin Y et al: Stress-dependent regulation of FOXO transcription factors by the SIRT1 deacetylase. Science 303: 2011-2015, 2004.

15. Liszt G, Ford E, Kurtev M and Guarente L: Mouse Sir2 homolog SIRT6 is a nuclear ADP-ribosyltransferase. J Biol Chem 280: 21313-21320, 2005.

16. Sauve AA and Schramm VL: SIR2: the biochemical mechanism of NAD(+)-dependent protein deacetylation and ADP-ribosyl enzyme intermediates. Curr Med Chem 11: 807-826, 2004.

17. Tanny JC, Dowd GJ, Huang J, Hilz H and Moazed D: An enzymatic activity in the yeast Sir2 protein that is essential for gene silencing. Cell 99: 735-745, 1999.

18. Tanny JC and Moazed D: Coupling of histone deacetylation to NAD breakdown by the yeast silencing protein Sir2: evidence for acetyl transfer from substrate to an NAD breakdown product. Proc Natl Acad Sci USA 98: 415-420, 2001.

19. Tanner KG, Landry J, Sternglanz R and Denu JM: Silent information regulator 2 family of NAD-dependent histone/protein deacetylases generates a unique product, 1-O-acetyl-ADP-ribose. Proc Natl Acad Sci USA 97: 14178-14182, 2000.

20. Lin SJ, Kaeberlein M, Andalis AA, Sturtz LA, Defossez PA, Culotta VC, et al: Calorie restriction extends Saccharomyces cerevisiae lifespan by increasing respiration. Nature 418: 344-348, 2002

21. Lin SJ, Defossez PA and Guarente L: Requirement of NAD and SIR2 for life-span extension by calorie restriction in Saccharomyces cerevisiae. Science 289: 2126-2128, 2000.

22. North BJ and Verdin E: Sirtuins: Sir2-related NAD-dependent protein deacetylases. Genome Biol 5: 224.1-7, 2004.

23. Motta MC, Divecha N, Lemieux M, Kamel C, Chen D, Gu W, et al: Mammalian SIRT1 represses forkhead transcription factors. Cell 116: 551-563, 2004.

24. Luo J, Nikolaev AY, Imai S, Chen D, Su F, Shiloh A, et al: Negative control of p53 by Sir2alpha promotes cell survival under stress. Cell 107: 137-148, 2001.
25. Vaziri H, Dessain SK, Ng Eaton E, Imai SI, Frye RA, Pandita TK, et al: hSIR2(SIRT1) functions as an NAD-dependent p53 deacetylase. Cell 107: 149-159, 2001.

26. Nemoto S, Fergusson MM and Finkel T: Nutrient availability regulates SIRT1 through a forkhead-dependent pathway. Science 306: 2105-2108, 2004.

27. Cohen HY, Miller C, Bitterman KJ, Wall NR, Hekking B, Kessler B, et al: Calorie restriction promotes mammalian cell survival by inducing the SIRT1 deacetylase. Science 305: 390-392, 2004.

28. Starai VJ, Celic I, Cole RN, Boeke JD and Escalante-Semerena JC: Sir2-dependent activation of acetyl-CoA synthetase by deacetylation of active lysine. Science 298: 2390-2392, 2002.

29. North BJ, Marshall BL, Borra MT, Denu JM and Verdin E: The human Sir2 ortholog, SIRT2, is an NAD ${ }^{+}$-dependent tubulin deacetylase. Mol Cell 11: 437-444, 2003.

30. Dryden SC, Nahhas FA, Nowak JE, Goustin AS and Tainsky MA: Role for human SIRT2 NAD-dependent deacetylase activity in control of mitotic exit in the cell cycle. Mol Cell Biol 23: 3173-3185, 2003

31. Birnboim HC and Doly J: A rapid alkaline extraction procedure for screening recombinant plasmid DNA. Nucleic Acids Res 7: $1513-1523,1979$

32. Mahlknecht U, Hoelzer D and Bucala R: Sequencing of genomic DNA. Biotechniques 27: 406-408, 1999.

33. Altschul SF, Madden TL, Schaffer AA, Zhang J, Zhang Z, Miller W, et al: Gapped BLAST and PSI-BLAST: a new generation of protein database search programs. Nucleic Acids Res 25: 3389-3402, 1997.

34. Wilbur WJ and Lipman DJ: Rapid similarity searches of nucleic acid and protein data banks. Proc Natl Acad Sci USA 80: 726-730, 1983.

35. Needleman SB and Wunsch CD: A general method applicable to the search for similarities in the amino acid sequence of two proteins. J Mol Biol 48: 443-453, 1970.

36. Thompson JD, Higgins DG and Gibson TJ: CLUSTAL W: improving the sensitivity of progressive multiple sequence alignment through sequence weighting, position-specific gap penalties and weight matrix choice. Nucleic Acids Res 22: 4673-4680, 1994.

37. Feng DF and Doolittle RF: Progressive sequence alignment as a prerequisite to correct phylogenetic trees. J Mol Evol 25: 351-360, 1987.

38. Fitzgerald $M$ and Shenk T: The sequence 5'-AAUAAA-3'forms parts of the recognition site for polyadenylation of late SV40 mRNAs. Cell 24: 251-260, 1981 .

39. Yang YH, Chen YH, Zhang CY, Nimmakayalu MA, Ward DC and Weissman S: Cloning and characterization of two mouse genes with homology to the yeast Sir2 gene. Genomics 69: 355-369, 2000.

40. Su AI, Cooke MP, Ching KA, Hakak Y, Walker JR, Wiltshire T, et al: Large-scale analysis of the human and mouse transcriptomes. Proc Natl Acad Sci USA 99: 4465-4470, 2002.

41. Su AI, Wiltshire T, Batalov S, Lapp H, Ching KA, Block D, et al: A gene atlas of the mouse and human protein-encoding transcriptomes. Proc Natl Acad Sci USA 101: 6062-6067, 2004.

42. Walker JR, Su AI, Self DW, Hogenesch JB, Lapp H, Maier R, Hoyer D and Bilbe G: Applications of a rat multiple tissue gene expression data set. Genome Res 14: 742-749, 2004.

43. Singer MF, Thayer RE, Grimaldi G, Lerman MI and Fanning TG: Homology between the KpnI primate and BamH1 (M1F-1) rodent families of long interspersed repeated sequences. Nucleic Acids Res 11: 5739-5745, 1983.

44. Lewin B: Regulation of transcription. Oxford University Press, New York, 1997.

45. Mitelman F, Mertens F and Johansson B: A breakpoint map of recurrent chromosomal rearrangements in human neoplasia. Nat Genet: 417-474, 1997.

46. Mahlknecht U, Ottmann OG and Hoelzer D: When the band begins to play: Histone acetylation caught in the crossfire of gene control. Mol Carcinog 27: 268-271, 2000. 